\title{
Persistent currents in mesoscopic Fibonacci rings
}

\author{
G. J. Jin \\ Department of Physics and National Laboratory of Solid State Microstructures, \\ Nanjing University, Nanjing 210093, People's Republic of China \\ Z. D. Wang \\ Department of Physics, The University of Hong Kong, Pokfulam Road, Hong Kong \\ A. Hu and S. S. Jiang \\ Department of Physics and National Laboratory of Solid State Microstructures, \\ Nanjing University, Nanjing 210093, People's Republic of China
}

(Received 8 October 1996)

\begin{abstract}
In the framework of a tight-binding model, we study energy spectra and persistent currents in mesoscopic Fibonacci rings threaded by a magnetic flux. It is found that the flux-dependent electron eigenenergies $E(\Phi)$ in mesoscopic Fibonacci rings still form "bands" with respect to the flux $\Phi$, but there is a scaling relation between the total "bandwidth" and the Fibonacci number. When the strength of the one-dimensional quasiperiodic potential increases, the persistent current decreases rapidly. Interestingly, for a generalized mixing model of mesoscopic Fibonacci rings, free-electron-like persistent current may appear if the number of electrons of the system takes a specific value. [S0163-1829(97)08816-4]
\end{abstract}

In recent years, there has been considerable interest in the persistent currents in one-dimensional metal or semiconductor mesoscopic rings threaded by a magnetic flux. ${ }^{1-4}$ An especially interesting fact is that there is a discrepancy about the magnitude of the persistent currents between theories and experiments. ${ }^{3,4}$ It is believed that disorder and interaction are two important factors that affect the amplitude of the current. Nevertheless, their influences have not been completely clarified. On the other hand, great experimental and theoretical efforts have been devoted to physical properties in onedimensional quasiperiodic structures. ${ }^{5-8}$ As is well known, the quasiperiodicity of Fibonacci structure has substantial effects on physical properties of condensed matter, and may provide a profound understanding of the intermediate region between periodic and random structures. Furthermore, a rational approximation is often employed in theoretical treatments of quasiperiodic behavior in Fibonacci chains or superlattices. Fortunately, this rational condition can be naturally realized in mesoscopic rings with the structure of Fibonacci chain. Such rings may be fabricated artificially. Therefore, it is worthwile to explore the properties of persistent currents in mesoscopic Fibonacci rings. In this paper, we first derive the basic formulas for persistent currents in mesoscopic Fibonacci rings; then the numerical results as well as relevant discussions are presented.

A Fibonacci chain is the simplest one-dimensional quasiperiodic structure, with two building units denoted by $A$ and $B$. Using these two units, a Fibonacci chain is formed according to the rule $S_{j+1}=\left\{S_{j}, S_{j-1}\right\}, S_{1}=A, S_{2}=A B$. For example, $S_{5}=A B A A B A B A$. It is reasonable to consider $A$ and $B$ as two kinds of site energies or hopping integrals. ${ }^{5} \mathrm{~A}$ mesoscopic Fibonacci ring is constructed by a finite Fibonacci chain with $N$ sites. For convenience, we assume that $N=F_{j}$, where $j$ is a generation number, and $F_{j}$ is a Fibonacci number obeying the recursion relation $F_{j}=F_{j-2}$ $+F_{j-1}$ with $F_{1}=F_{0}=1$. For a mesoscopic ring, it may be reasonable to consider $F_{j} \sim 10^{3}$.
In the tight-binding approximation, the Schrödinger equation for an electron in a one-dimensional mesoscopic Fibonacci ring reads

$$
t_{l+1} \psi_{l+1}+v_{l} \psi_{l}+t_{l} \psi_{l-1}=E \psi_{l},
$$

where $l$ is the site index, $t_{l}$ the hopping integral, and $v_{l}$ the on-site energy. For simplicity and without loss of generality, the spin freedom is not taken into account in the present paper. There are two simple types of one-dimensional quasiperiodic models. One is a transfer model, in which $v_{l}$ is taken to be constant, but $t_{l}$ is chosen to be two values $t_{A}$ and $t_{B}$ in a Fibonacci sequence. The other is an on-site model, in which $t_{l}$ is constant, but $v_{l}$ is set to be $v_{A}$ and $v_{B}$ in the Fibonacci sequence. In addition, there is a mixing model, in which both $v_{l}$ and $t_{l}$ take two values. For concreteness, we consider the on-site model first, in which two parameters $v_{A}$ and $v_{B}$ represent the quasiperiodic potential experienced by an electron in a mesoscopic Fibonacci ring.

Since $t_{l}$ is a constant, we can take its absolute value as an energy unit, or simply define $t_{l}=-1$. In the on-site model, Eq. (1) can be transformed into a matrix form as

$$
\left(\begin{array}{c}
\psi_{l+1} \\
\psi_{l}
\end{array}\right)=T_{l+1, l}\left(\begin{array}{c}
\psi_{l} \\
\psi_{l-1}
\end{array}\right),
$$

where

$$
T_{l+1, l}=\left(\begin{array}{cc}
-\left(E-v_{l}\right) & -1 \\
1 & 0
\end{array}\right) .
$$

When the ring is threaded by a magnetic flux $\Phi$, which leads to the twisted boundary conditions for the wave functions of the electrons in the ring, ${ }^{1}$ the equation for the global transfer matrix can be written as 


$$
\left(\begin{array}{c}
\psi_{N+1} \\
\psi_{N}
\end{array}\right)=M_{j}\left(\begin{array}{l}
\psi_{1} \\
\psi_{0}
\end{array}\right)=e^{i 2 \pi \Phi / \Phi_{0}}\left(\begin{array}{l}
\psi_{1} \\
\psi_{0}
\end{array}\right),
$$

where $\Phi_{0}=h c / e$ is the flux quantum, and $M_{j}$ $=\Pi_{l=1}^{N} T_{l+1,1} \cdot T_{l+1, l}$ and $M_{j}$ are all unimodular. All physical quantities are periodic in $\Phi$ with the period $\Phi_{0}$.

Denoting $\chi_{j}=(1 / 2) \operatorname{Tr} M_{j}$, the flux-dependent energy spectra of an electron in the mesoscopic Fibonacci ring can be obtained from the equation as

$$
\chi_{j}=\cos \left(2 \pi \Phi / \Phi_{0}\right) .
$$

The persistent current contributed from the $n$th level is

$$
I_{n}(\Phi)=-c \frac{\partial E_{n}(\Phi)}{\partial \Phi}=\frac{2 \pi c}{\Phi_{0}} \frac{\sin \left(2 \pi \Phi / \Phi_{0}\right)}{\partial \chi_{j} / \partial E_{n}} .
$$

Here $\chi_{j}$ and $\partial \chi_{j} / \partial E$ can be evaluated recursively. The useful recursion relations are

$$
\chi_{j}=2 \chi_{j-1} \chi_{j-2}-\chi_{j-3}
$$

and

$$
\frac{\partial \chi_{j}}{\partial E}=2\left(\chi_{j-1} \frac{\partial \chi_{j-2}}{\partial E}+\frac{\partial \chi_{j-1}}{\partial E} \chi_{j-2}\right)-\frac{\partial \chi_{j-3}}{\partial E},
$$

with the initial conditions

$$
\begin{aligned}
& \chi_{1}=-\left(E-v_{A}\right) / 2, \quad \chi_{0}=-\left(E-v_{B}\right) / 2, \quad \chi_{-1}=1, \\
& \partial \chi_{1} / \partial E=-\frac{1}{2}, \quad \partial \chi_{0} / \partial E=-\frac{1}{2}, \quad \partial \chi_{-1} / \partial E=0 .
\end{aligned}
$$

To calculate the charge stiffness later, we need also to evaluate the second-order derivatives

$$
\begin{aligned}
\frac{\partial^{2} E}{\partial\left(\Phi / \Phi_{0}\right)^{2}}= & -4 \pi^{2}\left(\frac{\partial \chi_{j}}{\partial E}\right)^{-3}\left[\cos \left(2 \pi \frac{\Phi}{\Phi_{0}}\right)\left(\frac{\partial \chi_{j}}{\partial E}\right)^{2}\right. \\
& \left.+\sin ^{2}\left(2 \pi \frac{\Phi}{\Phi_{0}}\right) \frac{\partial^{2} \chi_{j}}{\partial E^{2}}\right] .
\end{aligned}
$$

The corresponding recursion relation is found to be

$$
\begin{aligned}
\frac{\partial^{2} \chi_{j}}{\partial E^{2}}= & 2\left(\chi_{j-1} \frac{\partial^{2} \chi_{j-2}}{\partial E^{2}}+\frac{\partial^{2} \chi_{j-1}}{\partial E^{2}} \chi_{j-2}+2 \frac{\partial \chi_{j-1}}{\partial E} \frac{\partial \chi_{j-2}}{\partial E}\right) \\
& -\frac{\partial^{2} \chi_{j-3}}{\partial E^{2}}
\end{aligned}
$$

with the initial conditions

$$
\partial^{2} \chi_{1} / \partial E^{2}=0, \quad \partial^{2} \chi_{0} / \partial E^{2}=0, \quad \partial^{2} \chi_{-1} / \partial E^{2}=0 .
$$

The first nonzero term of the second derivatives is $\partial^{2} \chi_{2} / \partial E^{2}=1$. Equations (5)-(10) are our main analytical results, which will be used in numerical calculations. These equations are also suitable for the transfer model or even for the mixing model, provided the initial conditions are modified.

In the following numerical calculations, we set $v_{A}=-v_{B}=v$. Obviously, the parameter $v$ represents the strength of the quasiperiodicity. Once the Fibonacci generation number is chosen to be $j$, the total number of energy levels is $F_{j}$ for any fixed flux. At zero temperature, electrons

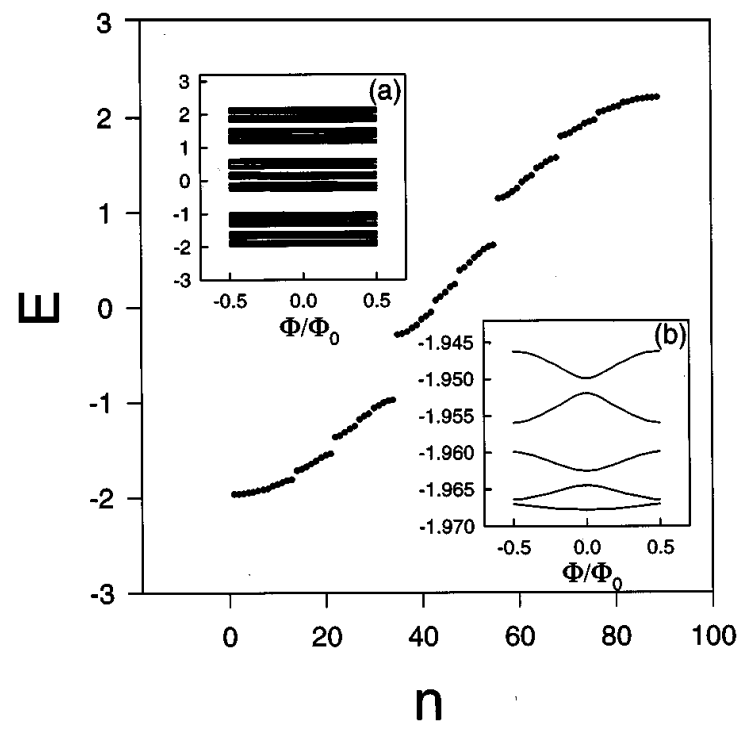

FIG. 1. The energy eigenvalues of a mesoscopic Fibonacci ring for the on-site model, where $j=10(N=89), v=0.5$, and $\Phi / \Phi_{0}=0.25$. The inset (a) shows the flux-dependent energy spectra for $-1 / 2<\Phi / \Phi_{0}<1 / 2$. The inset (b) shows the five lowest-energy "bands." All energies are in unit of $\left|t_{l}\right|$.

will occupy the levels one by one from the lowest level. If the number of electrons of the system is $N_{e}$, which is equal to the highest occupied-level index $m$, the energy of the system is given by

$$
E(\Phi)=\sum_{n=1}^{m} E_{n}(\Phi)
$$

and the total persistent current of the system is

$$
I(\Phi)=\sum_{n=1}^{m} I_{n}(\Phi) .
$$

Since the persistent current in a mesoscopic ring is completely determined by flux-dependent energy spectra of the system, we address it at first. Figure 1 shows the numerical results of the energy spectra for $j=10$ and $v=0.5$. At a fixed flux, for example $\Phi=0.25 \Phi_{0}$, there are $F_{10}=89$ eigenenergies. It can be seen clearly that they form a Cantor set. Actually, all $E_{n} \sim \Phi$ curves form three subband structures, which have been shown in inset (a) of Fig. 1. This characteristics is quite similar to those for the electrons, phonons, and spin waves in Fibonacci chain or superlattices. ${ }^{5-7}$ As pointed out by Büttiker and co-workers, ${ }^{1}$ there is a correspondence between an electron in a small normal onedimensional ring and an electron in a periodic potential, so flux-dependent electronic eigenenergies form "band" structures with respect to $\Phi$. In the mesoscopic Fibonacci rings, this conclusion is still correct. To see the $E_{n}(\Phi)$ curves clearly, the five lowest $E_{n}(\Phi)$ curves are plotted in inset (b) of Fig. 1. Every $E_{n}(\Phi)$ curve changes smoothly with flux. However, the quasiperiodicity affects the energy spectra of a mesoscopic Fibonacci ring significantly. One example is that the total "bandwidth" $B \quad\left[=\Sigma_{n} \mid E_{n}\left(\Phi / \Phi_{0}=0\right)\right.$ $\left.-E_{n}\left(\Phi / \Phi_{0}=\frac{1}{2}\right) \mid\right]$ diminishes rapidly with increasing Fibonacci generation number $j$. There exists a scaling relation between $B$ and $F_{j}$, i.e., $B \sim F_{j}^{-\alpha}$, as shown in Fig. 2 . The 


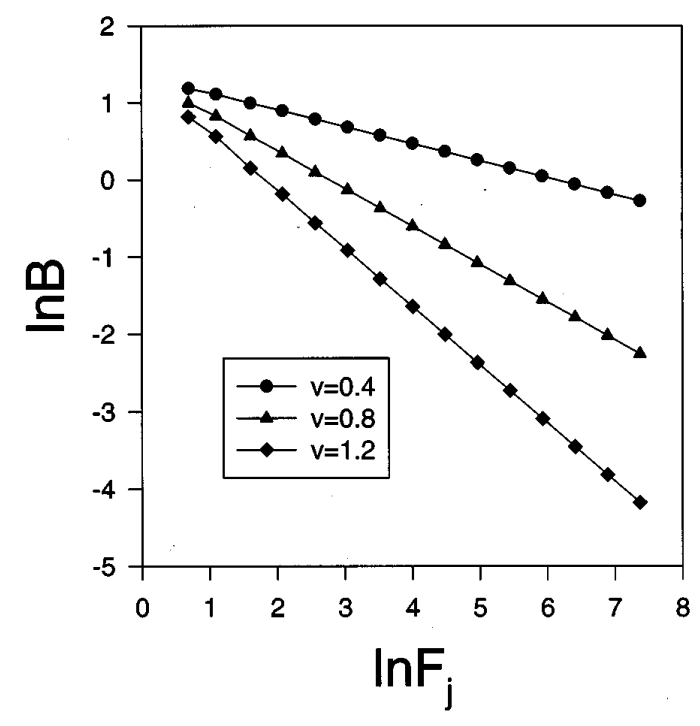

FIG. 2. Log-log plot of the total "bandwidth" $B$ and the Fibonacci number $F_{j} . B$ is also in unit of $\left|t_{l}\right|$.

power index $\alpha$ depends on $v$. For $v=0.4,0.8$, and 1.2, $\alpha=0.22,0.49$, and 0.75 , respectively. Although the scaling index $\alpha$ may be quite different, this scaling behavior is similar to those in other quasiperiodic systems, ${ }^{9}$ as the common origin of the scaling comes from the quasiperiodicity of the structure.

The persistent current in a mesoscopic ring is obtained from the energy spectra in terms of Eqs. (6) and (12). Usually, the narrower the "bandwidth" is, the smaller the maximum persistent current. Here let us consider a case with even number of electrons and near half-filled, where $j=12$ $\left(N=F_{j}=233\right)$ and $N_{e}=116$. When $v=0$, the relationship between the persistent current and the magnetic flux is freeelectron behavior. As $v$ increases, $I(\Phi)$ is suppressed, as shown in Fig. 3, where the maximum persistent current of the periodic tight-binding model $I_{0}=(4 \pi c /$ $\left.N \Phi_{0}\right) \sin \left(N_{e} \pi / N\right)$ is taken to be the current unit. To see the

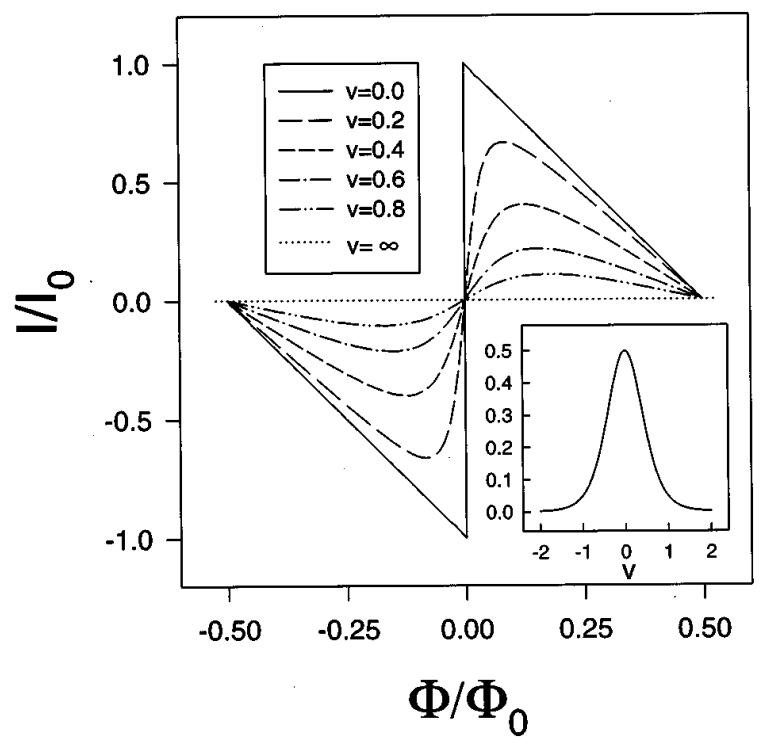

FIG. 3. The persistent current $I$ vs flux $\Phi$ for various on-site energies, where $j=12(N=233), N_{e}=116$. The inset gives the dependence of $I$ on the on-site energy $v$ at $\Phi / \Phi_{0}=0.25$. suppression clearly, we plot $I$ against $v$ at a fixed $\Phi$ in the inset of Fig. 3. Here the overall behavior of $I(\Phi)$ is somewhat similar to that in a disordered mesoscopic ring. ${ }^{2}$ But, unlike disordered systems, the mesoscopic Fibonacci ring is deterministic and no average procedure needs to be taken. In Fig. 4 , we show the results for an odd $N_{e}$ case. One can find that $I \sim \Phi$ curves shift $\Phi / \Phi_{0}=\frac{1}{2}$ along the flux axis when compared with that for even $N_{e}$ in Fig. 3, as in the periodic systems. ${ }^{10}$ Also, we calculate the charge stiffness, which represents the response of the persistent current to the applied flux, and is defined as ${ }^{11}$

$$
D=\frac{F_{j}}{4 \pi^{2}} \frac{\partial^{2} E(\Phi)}{\partial\left(\Phi / \Phi_{0}\right)^{2}} .
$$

By combining Eqs. (9) and (10) with Eq. (13), the numerical results of $D$ for $\Phi / \Phi_{0}=0,0.3,0.35$, and 0.4 are plotted, as shown in the inset of Fig. 4. We can see that, as $v$ increases, the response of the persistent current to the applied flux is more and more difficult for $\Phi<0.35$; while for 0.35 $<\Phi / \Phi_{0}<0.5$, the situation becomes a little bit complicated. When $v$ is significantly large, $|D|$ vanishes for all $\Phi$. When $v \rightarrow 0, D$ approaches 0.636 , which can be analytically derived from the energy spectra formulas of the tight-binding periodic mesoscopic ring. Similar behaviors can also be found for even-number electrons, but with $\Phi$ being shifted by $\Phi_{0} / 2$.

The transfer model can be treated in a similar way. In fact, the main results are almost the same. Here we wish to address further an issue of higher transmission and extended states in the mixing model. As in a generalized Fibonacci chain, ${ }^{8}$ we need to take into account three reduced energy parameters: the on-site energy $v$, the hopping integral $t$, and the electron energy $E$, where $t=t_{A A} / t_{A B}$ with $t_{A A}$ and $t_{A B}$ as the hopping integrals between corresponding sites $\left(t_{A B}\right.$ is taken as the energy unit). In this model, there may exist extended states, as in other quasiperiodic and disordered

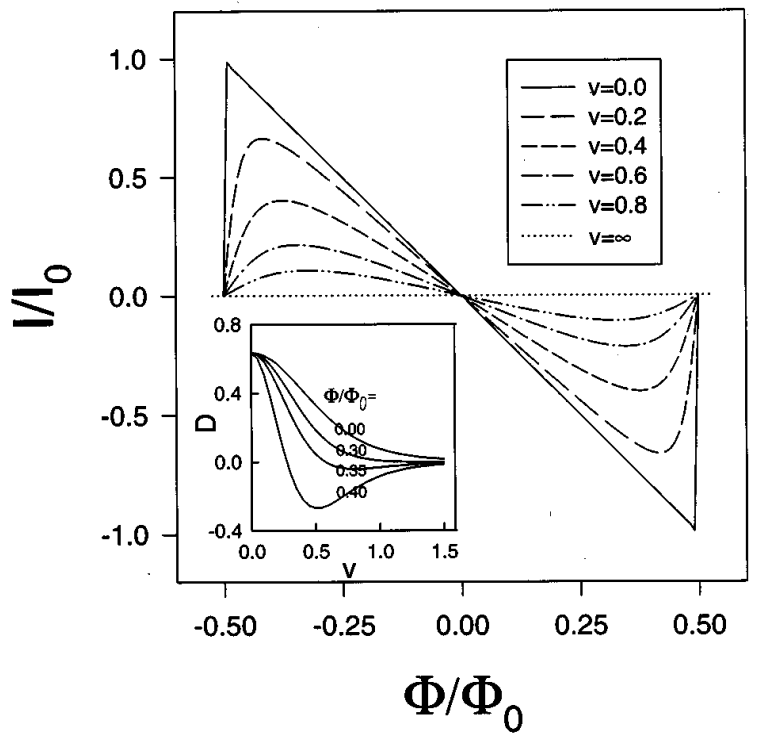

FIG. 4. The persistent current $I$ vs flux $\Phi$ for various on-site energies, where $j=12$, and $N_{e}=117$. The inset shows the variation of the charge stiffness with the on-site energy $v$ at $\Phi / \Phi_{0}=0,0.30,0.35$, and 0.40 , respectively. 

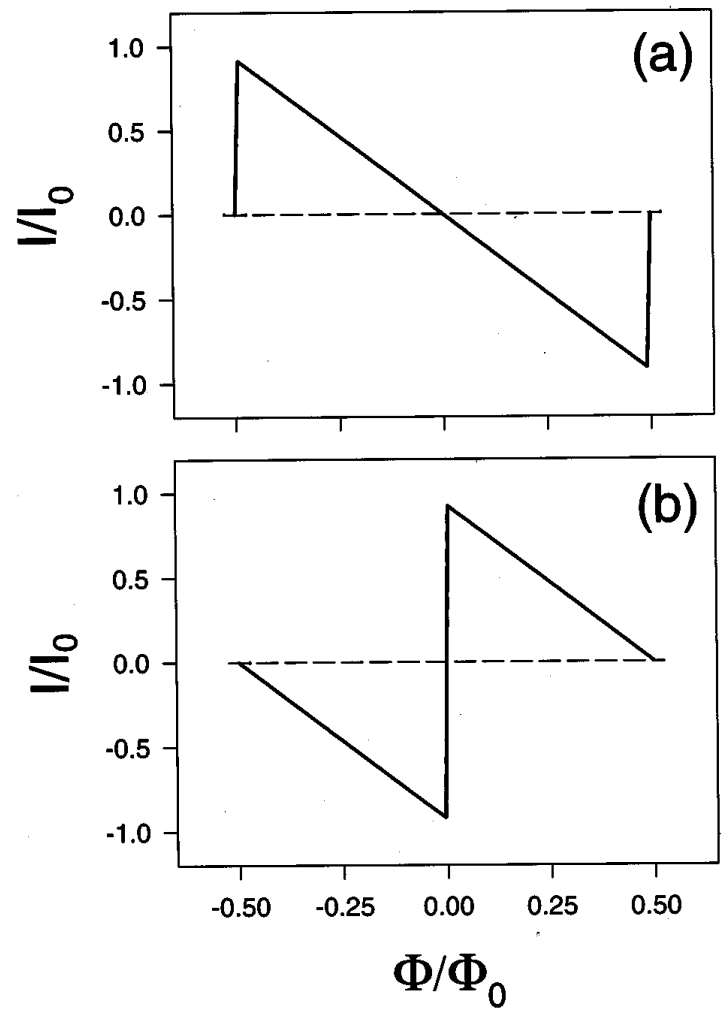

FIG. 5. The persistent current $I$ as a function of $\Phi$ in the mixing model, where $t=2, v=0.6$. (a) $N_{e}=85$. (b) $N_{e}=86$.

systems, ${ }^{12}$ which correspond to higher transmission coefficients. If the three energy parameters satisfy a relation ${ }^{8}$

$$
E_{n}=v\left[\left(1+t^{2}\right) /\left(1-t^{2}\right)\right],
$$

we can show that the global transfer matrix of the system $M_{j}=R_{C}^{N}$, where

$$
R_{C}=\left(\begin{array}{cc}
-t^{-1}\left(E_{n}-v\right) & -t \\
t^{-1} & 0
\end{array}\right) .
$$

In terms of Chebyshev polynomials of the second order, we have

$$
M_{j}=\frac{1}{\sin \theta}\left(\begin{array}{cc}
\sin [(N+1) \theta] & -t \sin (N \theta) \\
t^{-1} \sin (N \theta) & -\sin [(N-1) \theta]
\end{array}\right),
$$

where $\cos \theta \equiv-(1 / 2 t)\left(E_{n}-v\right)$. From Eq. (16), it is straightforward to show that $\chi_{j}=\cos (N \theta)$. Thus, from Eq. (5), we are able to have $\theta=(2 \pi / N)\left(n+\Phi / \Phi_{0}\right)$. If $E_{n}$ is the highest energy level (i.e., $n=m$ ), Eq. (6) leads to

$$
I_{m}(\Phi)=\frac{4 \pi c}{N} \sin \left[\frac{2 \pi}{N}\left(m+\frac{\Phi}{\Phi_{0}}\right)\right]
$$

which is just the result of a periodic tight-binding model. Since the $I_{m}(\Phi)$ is the dominant term in $I(\Phi),{ }^{13}$ a freeelectron-like persistent current is naturally expected. Therefore, if the electrons are appropriately accommodated in the energy levels such that $E_{m}$ satisfies Eq. (14) approximately, the persistent current is very close to the free-electron case [see the numerical results calculated from Eqs. (6) and (12) in Fig. 5], and the transmission coefficients can be quite large or even close to 1 . For example, when $t=2, v=0.6$, and $E=-1$ from Eq. (14), the transmission coefficient $\tau=0.9968 .{ }^{14}$ For $j=12$, the energies of the 85th and 86th energy bands are very close to -1 . The deviation of the energy from $E_{n}=-1$ is less than $5 \%$ as $\Phi$ changes.

In summary, we have investigated the persistent currents in mesoscopic Fibonacci rings. The effects of quasiperiodicity on the energy spectra as well as on the persistent currents have been elucidated.

This work was supported by Hong Kong RGC Grant No. HKU262/95P, the National Natural Science Foundation of China, and the Provincial Natural Science Foundation of Jiangsu.
${ }^{1}$ M. Büttiker et al., Phys. Lett. 96A, 365 (1983); R. Landauer and M. Büttiker, Phys. Rev. Lett. 54, 2049 (1985).

${ }^{2}$ H. F. Cheung et al., Phys. Rev. B 37, 6050 (1988).

${ }^{3}$ L. P. Lévy et al., Phys. Rev. Lett. 64, 2074 (1990); V. Chandrasekhar et al., ibid. 67, 3578 (1991); D. Mailly et al., ibid. 70, 2020 (1993).

${ }^{4}$ V. Ambegaokar and U. Eckern, Phys. Rev. Lett. 65, 381 (1990); F. von Oppen and E. K. Riedel, ibid. 66, 84 (1991); B. L. Altshuler et al., ibid. 66, 88 (1991); M. Abraham and R. Berkovits, ibid. 70, 1509 (1993); G. Bouzerar et al., Phys. Rev. B 49, 8258 (1994).

${ }^{5}$ M. Kohmoto et al., Phys. Rev. Lett. 50, 1870 (1983); Phys. Rev. B 35, 1020 (1987)

${ }^{6}$ R. Merlin et al., Phys. Rev. Lett. 55, 1768 (1985); P. Hawrylak and J. J. Quinn, ibid. 57, 380 (1986); B. L. Johnson and R. E. Camley, Phys. Rev. B 44, 1225 (1991).

${ }^{7}$ J. W. Feng et al., Phys. Rev. B 52, 15312 (1995); G. J. Jin et al., ibid. 54, 1883 (1996).

${ }^{8}$ V. Kumar, J. Phys. Condens. Matter 2, 1349 (1990).

${ }^{9}$ M. Kohmoto, Phys. Rev. Lett. 51, 1198 (1983); P. Hawrylak and
J. J. Quinn, ibid. 57, 380 (1986); T. Janssen and M. Kohmoto, Phys. Rev. B 38, 5811 (1988).

${ }^{10}$ Z. D. Wang and Jian-Xin Zhu, Phys. Rev. B 52, 5275 (1995); Jian-Xin Zhu et al., ibid. 52, 14505 (1995).

${ }^{11}$ G. Bouzerar et al., Phys. Rev. B 49, 8258 (1994); T. Giamarchi and B. S. Shastry, ibid. 51, 10915 (1995).

${ }^{12}$ V. Kumar and G. Ananthakrishna, Phys. Rev. Lett. 59, 1476 (1987); D. H. Dunlap et al., ibid. 65, 88 (1990).

${ }^{13}$ For various quasiperiodic strengths $v$, we have calculated the current of the highest occupied level $I_{m}(\Phi)$ and the total current $I(\Phi)$, and find that $I_{m}(\Phi)$ is dominant in the $I(\Phi)$, which is due to the fact that the contributions from the levels of $n$ and $n+1$ $(n<m$ and $m \gg 1)$ cancel each other, as in the periodic mesoscopic ring.

${ }^{14}$ If we consider $E, v$, and $t$ as three independent parameters, and denote the global transfer matrix as $M(N)$ with matrix elements $m_{i, j} s \quad(i, j=1,2)$, where $m_{i, j}$ is a function of the three energy parameters, the transmission coefficient can be obtained as

$$
\tau(N)=\frac{4-E^{2}}{\left[t_{21}-t_{12}+\left(t_{22}-t_{11}\right) E / 2\right]^{2}+\left(t_{22}+t_{11}\right)^{2}\left(1-E^{2} / 4\right)} .
$$

